Revista de Matemática: Teoría y Aplichciones 4(2): 25-42 (1997)

\title{
ALGORITMOS DE REDUCCIÓN PARA BIGRÁFICAS DIFERENCIALES
}

\author{
JuAN BOZA CORDERO ${ }^{1}$
}

\begin{abstract}
Resumen
Se estudian tres algoritmos de reducción para bigráficas diferenciales: regularización, eliminación de objetos y reducción de una flecha. En cada caso se determina la bigráfica resultante y la relación entre las respectivas categorías de representaciones. Estos algoritmos se aplican luego a las bigráficas 'schurian'.
\end{abstract}

\section{Introducción}

En la demostración del famoso teorema 'manso-salvaje' de Drozd (toda álgebra de dimensión finita sobre un campo algebraicamente cerrado es mansa o salvaje, y no de ambos tipos a la vez), se aplicaron de manera decisiva los algoritmos de reducción de la teoría de representaciones de categorías graduadas diferenciales $\left[\mathrm{D}_{1}, \mathrm{D}_{2}, \mathrm{CB}_{1}, \mathrm{M}\right]$. Los algoritmos se establecieron a principios de los setenta en el contexto de ciertos problemas matriciales, utilizándose el punto de vista de las bigráficas diferenciales, formalizadas más adelante en la teoría de representaciones de categorías graduadas diferenciales [RK]. Para fundamentar de modo más adecuado algunos aspectos de dicha teoría, fueron introducidos los 'bocses' (siglas de: 'bimodule over a category with coalgebra structure') [RK, Ro], que han mostrado ser una estructura adecuada y con muchas posibilidades de desarrollo [Bo, $\left.\mathrm{BZ}, \mathrm{CB}_{1}, \mathrm{CB}_{2}, \mathrm{CB}_{3}, \mathrm{D}_{3}, \mathrm{O}\right]$.

La tendencia actual es a utilizar el aparato formal de los bocses para presentar los resultados, aunque se continúa pensando con frecuencia en los términos más intuitivos de las bigráficas. El hecho fundamental es que ambos enfoques son equivalentes, como se estableció en [Bo]. En el presente trabajo se escogió operar directamente en el contexto de las bigráficas diferenciales, exponiendo con detenimiento tres algoritmos (regularización, eliminación de objetos y reducción de una flecha), que en la última sección son utilizados para efectuar un estudio de las bigráficas 'schurian', como ejemplo de aplicación interesante de la teoría.

\footnotetext{
${ }^{1}$ Centro de Investigaciones en Matemáticas Puras y Aplicadas (Cimpa), Escuela de Matemática, Universidad de Costa Rica, 2060 San José, Costa Rica
} 


\section{Representaciones de bigráficas diferenciales}

En lo que sigue $k$ denota un campo.Por definición, una bigráfica $\mathcal{B}$ es una gráfica finita orientada con dos tipos de flechas: unas de grado 0, dibujadas con un trazo continuo, $i \longrightarrow j$; y otras de grado 1, dibujadas con un trazo discontinuo: $i--\rightarrow j$. El álgebra de caminos $k \mathcal{B}$ es entonces un álgebra graduada, con la graduación inducida por el grado de las flechas. Así,

$$
k \mathcal{B}=(k \mathcal{B})_{0} \oplus(k \mathcal{B})_{1} \oplus \cdots
$$

Una bigráfica diferencial es un triple $(\mathcal{B}, \delta, k)$, en donde $\mathcal{B}$ es una bigráfica y $\delta$ : $k \mathcal{B} \longrightarrow k \mathcal{B}$ una transformación lineal, sujeta a las siguientes condiciones:

(i) si $a$ es homogéneo de grado $i(i=0,1)$, entonces $\delta(a)$ tiene grado $i+1$;

(ii) si $a, b$ son homogéneos, $\delta(a b)=\delta(a) b+(-1)^{g r(a)} a \delta(b)$;

(iii) para todo camino trivial $e, \delta(e)=0$;

(iv) $\delta^{2}=0$

Unas palabras sobre la notación: la diferencial de una flecha $a: i \longrightarrow j$ de grado 0 se escribe

$$
\delta(a)=\sum_{l} b_{l} x_{l} c_{l}
$$

en donde $b_{l}, c_{l}$ son caminos de grado $0, \mathrm{y} x_{l}$ flechas de grado 1 . Asimismo, para una flecha $x: i--\rightarrow j$ de grado 1 , se denota

$$
\delta(x)=\sum_{s} x_{s_{2}} x_{s_{1}}
$$

en donde $x_{s_{2}}, x_{s_{1}}$ son caminos de grado 1 . La bigráfica $\mathcal{B}$ posee un carcaj pleno, determinado por las flechas de grado 0 , y denotado $\mathcal{B}_{0}$. La correspondiente álgebra de caminos $k \mathcal{B}_{0}$, se denotará por $A=k \mathcal{B}_{0}$. Es claro que $A=(k \mathcal{B})_{0}$, el primer sumando directo de (1). Como al multiplicar un camino de grado 1, por la derecha o por la izquierda, por un camino de grado 0, el camino original preserva su grado, $(k \mathcal{B})_{1}$ es un $A-A$-bimódulo.

Para empezar con la teoría de representaciones, en primer lugar se define una representación de la bigráfica diferencial $(\mathcal{B}, \delta, k)$, como una $k$-representación del carcaj $\mathcal{B}_{0}$. Los morfismos sí se definen de manera distinta. De hecho, si $M, N$ son representaciones de la bigráfica diferencial $(\mathcal{B}, \delta, k)$, cuyos vértices son $1,2, \ldots, n$, un morfismo $f: M \longrightarrow N$ está dado por una familia doble de transformaciones lineales:

$$
f=\left(f_{i}^{0} ; f^{1}(x)\right)
$$

en donde $1 \leq i \leq n$, y $x$ recorre el conjunto de las flechas de grado 1 . Aquí, $f_{i}^{0}: M_{i} \longrightarrow N_{i}$; y si $x: i--\rightarrow j$, entonces $f^{1}(x): M_{i} \longrightarrow N_{j}$. Ha de cumplirse la siguiente condición para toda flecha $a: i \longrightarrow j$ de grado 0 , con diferencial (2):

$$
f_{j}^{0} M(a)-N(a) f_{i}^{0}=\sum_{l} N\left(b_{l}\right) f^{1}\left(x_{l}\right) M\left(c_{l}\right) .
$$

Para el lector será útil dibujar el diagrama correspondiente a la situación anterior, y compararlo con el caso de morfismos de carcajes. 
Otro recurso notacional: el segundo miembro de (5) se escribe como $\sum_{l} N\left(b_{l}\right) f^{1}\left(x_{l}\right) M\left(c_{l}\right)=f^{1}(\delta(a))$. De manera general, para un camino arbitrario de grado $1, \gamma=b_{l} x_{l} c_{l}$, se define la transformación lineal $f^{1}(\gamma)=N\left(b_{l}\right) f^{1}\left(x_{l}\right) M\left(c_{l}\right)$.

Lo que se desea es definir la categoría [MacL] de las representaciones de la bigráfica diferencial; hasta ahora se tienen los objetos y los morfismos. Para continuar, dados morfismos $f: L \longrightarrow M, g: M \longrightarrow N$, con $f=\left(f_{i}^{0} ; f^{1}(x)\right), g=\left(g_{i}^{0} ; g^{1}(x)\right)$, su composición se define como el morfismo $g f: L \longrightarrow N$, tal que $g f=\left((g f)_{i}^{0},(g f)^{1}(x)\right)$, en donde $(g f)_{i}^{0}=g_{i}^{0} f_{i}^{0} ;$ y para toda flecha de grado $1, x: i--\rightarrow j$, con diferencial (3),

$$
(g f)^{1}(x)=g^{1}(x) f_{i}^{0}+g_{j}^{0} f^{1}(x)+\sum_{s} g^{1}\left(x_{s_{2}}\right) f^{1}\left(x_{s_{1}}\right)
$$

En este momento se requiere demostrar que la composición de morfismos está bien definida, es decir, que $g f: L \longrightarrow N$ verifica la condición (5). En efecto,

$$
\begin{aligned}
g_{j}^{0} f_{j}^{0} L(a)-N(a) g_{i}^{0} f_{i}^{0} & =g_{j}^{0}\left(M(a) f_{i}^{0}+f^{1} \delta(a)\right)-\left(g_{j}^{0} M(a)-g^{1}(\delta(a))\right) f_{i}^{0} \\
& =g_{j}^{0} f^{1} \delta(a)+g^{1}(\delta(a)) f_{i}^{0} \\
& =(g f)^{1}(\delta(a))
\end{aligned}
$$

puesto que $\delta^{2}=0$

Para toda representación $M$, el morfismo identidad, como se comprueba fácilmente, está dado por la familia doble: $i d_{M}=\left(i d_{M_{i}} ; 0, \ldots, 0\right)$.

La composición de morfismos es asociativa. En efecto, dados morfismos $f, g, h, L \stackrel{f}{\longrightarrow}$ $M \stackrel{g}{\longrightarrow} N \stackrel{h}{\longrightarrow} T$, es claro que: $[h(g f)]_{i}^{0}=[(h g) f]_{i}^{0}$. Sea ahora $x: i--\rightarrow j$ una flecha de grado 1 con diferencial (3), e $i--\stackrel{x_{s_{1}}}{\rightarrow} s_{1}--\rightarrow j$, con diferenciales

$$
\begin{aligned}
\delta\left(x_{s_{2}}\right) & =\sum_{r} x_{s, r_{2}} x_{s_{2}, r_{1}} \quad s_{1} \stackrel{x_{s_{2} r_{1}}}{\rightarrow} r_{1} \stackrel{x_{s_{2} r_{2}}}{\rightarrow} j, \\
\delta\left(x_{s_{1}}\right) & =\sum_{t} x_{s_{1} t_{2}} x_{s_{1}, t_{1}} \quad i \stackrel{x_{s_{1} t_{1}}}{\rightarrow} t_{1} \stackrel{x_{s_{1} t_{2}}}{\rightarrow} s_{1} .
\end{aligned}
$$

Aplicando (6) adecuadamente,

$$
\begin{aligned}
{[h(g f)]^{1}(x)=} & h_{j}^{0}(g f)^{1}(x)+h^{1}(x) g_{i}^{0} f_{i}^{0}+\sum_{s} h^{1}\left(x_{s_{2}}\right)(g f)^{1}\left(x_{s_{1}}\right) \\
= & h_{j}^{0}\left[g_{j}^{0} f^{1}(x)+g^{1}(x) f_{i}^{0}+\sum_{s} g^{1}\left(x_{s_{2}}\right) f^{1}\left(x_{s_{1}}\right)\right]+h^{1}(x) g_{i}^{0} f_{i}^{0}+ \\
& +\sum_{s} h^{1}\left(x_{s_{2}}\right)\left[g_{s_{1}}^{0} f^{1}\left(x_{s_{1}}\right)+g^{1}\left(x_{s_{1}}\right) f_{i}^{0}+\right. \\
& \left.+\sum_{t} g^{1}\left(x_{s_{1}, t_{2}}\right) f^{1}\left(x_{s_{1}, t_{1}}\right)\right] .
\end{aligned}
$$

Haciendo un desarrollo parecido a $(7)$ para $[(h g) f]^{1}(x)$, se ve que para obtener la igualdad: $[h(g f)]^{1}(x)=[(h g) f]^{1}(x)$, basta verificar que

$$
\sum_{s, t} h^{1}\left(x_{s_{2}}\right) g^{1}\left(x_{s_{1} t_{2}}\right) f^{1}\left(x_{s_{1} t_{1}}\right)=\sum_{s, r} h^{1}\left(x_{s_{2} r_{2}}\right) g^{1}\left(x_{s_{2} r_{1}}\right) f^{1}\left(x_{s_{1}}\right) .
$$


Para obtener (8), hace falta una pequeña disgresión. Para vértices $i, j$ cualesquiera, sea $V^{\prime}(i, j)$ el espacio generado por las flechas $i--\rightarrow j$ de grado 1 . Si se tienen vértices $i, j, l$, es fácil convencerse [Bo, Cap.1] de que existe una transformación lineal tal que:

$$
g^{1} \cdot f^{1}: V^{\prime}(j, l) \otimes_{k} V^{\prime}(i, j) \longrightarrow \operatorname{Hom}_{k}\left(L_{i}, N_{j}\right), y \otimes x \mapsto g^{1}(y) \circ f^{1}(x)
$$

para la cual vale la asociatividad : $h^{1} \cdot\left(g^{1} \cdot f^{1}\right)=\left(h^{1} \cdot g^{1}\right) \cdot f^{1}$. Utilizando el hecho de que $\delta^{2}(x)=0$, se obtiene: $\sum_{s} \delta\left(x_{s_{2}}\right) x_{s_{1}}=\sum_{s} x_{s_{2}} \delta\left(x_{s_{1}}\right)$, y de aquí,

$$
\sum_{s}\left(h^{1} \cdot g^{1}\right)\left(\delta\left(x_{s_{2}}\right)\right) f^{1}\left(x_{s_{1}}\right)=\sum_{s} h^{1}\left(x_{s_{2}}\right)\left(g^{1} \cdot f^{1}\right)\left(\delta\left(x_{s_{1}}\right)\right)
$$

que es la igualdad (8). Con lo anterior se ha demostrado entonces el resultado siguiente.

Teorema La familia de las representaciones de una bigráfica diferencial $(\mathcal{B}, \delta, k)$ constituye una categoría.

La categoría de las representaciones de $(\mathcal{B}, \delta, k)$ se denota por $\operatorname{rep}(\mathcal{B}, \delta, k)$, o simplemente $\operatorname{rep} \mathcal{B}$, si no hay posibilidad de confusión.

Las bigráficas que se utilizan en las aplicaciones son 'triangulares', concepto que se define enseguida. Una bigráfica diferencial $(\mathcal{B}, \delta, k)$ se llama triangular si sus flechas de grado 0 están ordenadas como $a_{1}<a_{2}<\cdots<a_{t}$, de modo tal que todo $\delta\left(a_{i}\right)=\sum_{l=1}^{r} b_{l} x_{l} c_{l}$ , con los $b_{l}, c_{l}$ caminos de grado 0 , compuestos por flechas del conjunto $\left\{a_{1}, \cdots, a_{i-1}\right\}$. Se pide también que exista un orden entre las flechas de grado 1, de manera que para toda flecha $x$ de grado $1, \delta(x)=\sum_{s} x_{s_{2}} x_{s_{1}}$, y en los caminos $x_{s_{2}}, x_{s_{1}}$ aparecen únicamente flechas menores que $x$.

Una propiedad importante de las bigráficas triangulares es que un morfismo $f: M \longrightarrow$ $N$ dado por (4) es un isomorfismo si, y solamente si, los $f_{i}^{0}$ son isomorfismos.

\section{3 Álgebras y bigráficas}

En esta sección se desarrollará un ejemplo de cómopuede transformarse el estudio de la categoría de las representaciones de un álgebra [AR, Ri], en el estudio de la categoría de las representaciones de una bigráfica diferencial. Esto se hace por la 'reducción de una flecha' del carcaj del álgebra, uno de los algoritmos de la teoría, que se examinará con detenimiento más adelante.

Sea $A=k Q$ el álgebra de caminos del carcaj:

$$
Q: 4 \stackrel{a}{\longleftarrow} 1 \stackrel{\alpha}{\longrightarrow} 2
$$

A continuación se reduce la flecha $\alpha$, acción que lleva a cambiar el carcaj $Q$ por una bigráfica diferencial $\mathcal{B}$, con la propiedad de que las estructuras $Q, \mathcal{B}$ poseen categorías de representaciones equivalentes, es decir, básicamente iguales. 
Un morfismo entre representaciones de $A=k Q, g: M \longrightarrow M^{\prime}$, en donde $M=$ $\left(M_{1}, M_{2}, M_{4}\right), M=\left(M_{1}^{\prime}, M_{2}^{\prime}, M_{4}^{\prime}\right)$, consta de transformaciones lineales $g_{1}, g_{2}, g_{4}$, tales que el siguiente diagrama conmuta:

$$
\begin{array}{cccccc}
M_{4} & \stackrel{M(a)}{\longleftarrow} & M_{1} & \stackrel{M(\alpha)}{\longrightarrow} & M_{2} & \\
g_{4} \downarrow & & g_{1} \downarrow & & \downarrow g_{2} & . \\
M_{4}^{\prime} & \stackrel{M^{\prime}(a)}{\longleftarrow} & M_{1}^{\prime} & \stackrel{M^{\prime}(\alpha)}{\longrightarrow} & M_{2}^{\prime} &
\end{array}
$$

Para aligerar la notación, se escribirá: $M(\alpha)=\alpha, M(a)=a$, etc. La conmutatividad del anterior diagrama se escribe entonces así:

$$
\left\{\begin{array}{c}
g_{4} a-a^{\prime} g_{1}=0 \\
g_{2} \alpha-\alpha^{\prime} g_{1}=0
\end{array} .\right.
$$

Se aprovechan ahora las descomposiciones:

$$
\begin{aligned}
& M_{1} \cong \operatorname{ker} \alpha \oplus i m \alpha, \quad M_{2} \cong i m \alpha \oplus W \\
& M_{1}^{\prime} \cong \operatorname{ker} \alpha^{\prime} \oplus i m \alpha^{\prime}, \quad M_{2}^{\prime} \cong i m \alpha^{\prime} \oplus W^{\prime}
\end{aligned}
$$

para escribir $g_{1}, g_{2}$ como matrices de bloques (con una notación que se mostrará como la adecuada más adelante):

$$
g_{1}=\left(\begin{array}{cc}
g_{1}^{0} & g^{1}(x) \\
0 & g_{3}^{0}
\end{array}\right) \quad, \quad g_{2}=\left(\begin{array}{cc}
g_{3}^{0} & g^{1}(y) \\
0 & g_{2}^{0}
\end{array}\right)
$$

en donde: $g_{1}^{0}: \operatorname{ker} \alpha \longrightarrow \operatorname{ker} \alpha^{\prime}, g_{2}^{0}: W \longrightarrow W^{\prime}, g_{3}^{0}: i m \alpha \longrightarrow i m \alpha^{\prime}$, $g^{1}(x): i m \alpha \longrightarrow \operatorname{ker} \alpha^{\prime}, g^{1}(y): W \longrightarrow i m \alpha^{\prime}$. Asimismo, se escribe $a=\left(a_{1}, a_{2}\right)$, con $a_{1}: \operatorname{ker} \alpha \longrightarrow M_{4}, a_{2}: i m \alpha \longrightarrow M_{4}$; de manera similar, $a^{\prime}=\left(a_{1}^{\prime}, a_{2}^{\prime}\right)$.

De la primera igualdad de (9) se obtienen las relaciones:

$$
\left\{\begin{array}{c}
g_{4} a_{1}-a_{1}^{\prime} g_{1}^{0}=0 \\
g_{4} a_{2}-a_{2}^{\prime} g_{3}^{0}=a_{1}^{\prime} g^{1}(x)
\end{array},\right.
$$

las cuales se disponen en el siguiente diagrama:

$$
\begin{aligned}
& \text { im } \alpha \\
& \downarrow g_{3}^{0} \\
& M_{4} \stackrel{a_{1}}{\longleftarrow} \operatorname{ker} \alpha \quad i m \alpha^{\prime} \stackrel{g^{1}(y)}{\longleftarrow} W \\
& g_{4} \downarrow \quad \downarrow g_{1}^{0} \quad \downarrow g_{2}^{0} \\
& M_{4}^{\prime} \stackrel{a_{1}^{\prime}}{\longleftarrow} \operatorname{ker} \alpha^{\prime} \quad W^{\prime}
\end{aligned}
$$

El diagrama (13) apunta de manera clara a la bigráfica diferencial:

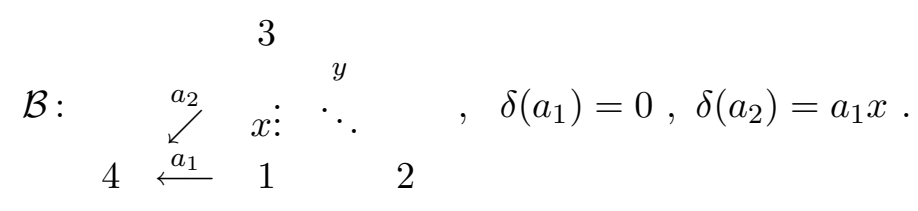


De hecho, existe una equivalencia[MacL] entre las categorías de representaciones del álgebra de caminos $A=k Q$ y las representaciones de la bigráfica $\mathcal{B}$,

$$
F: \operatorname{rep} \mathcal{B} \stackrel{\sim}{\longrightarrow} \bmod A
$$

que se explicitará a continuación.

Para definir el funtor $F$ en objetos, si se tiene $N \in$ rep $\mathcal{B}$, dada por $N=\left(N_{i}, 1 \leq i \leq 4 ; N\left(a_{j}\right), 1 \leq j \leq 2\right)$, se define la representación $M=F(N) \in \bmod A$ así: $M_{1}=N_{1} \oplus N_{3}, M_{2}=N_{3} \oplus N_{2}, M_{4}=N_{4}$. Asimismo, se definen las transformaciones $M(\alpha): M_{1} \longrightarrow M_{2}$, como $M(\alpha)=\left(\begin{array}{cc}0 & i d_{N_{3}} \\ 0 & 0\end{array}\right)$,

$M(a): M_{1}=N_{1} \oplus N_{3} \longrightarrow M_{4}$, como $M(a)=\left(N\left(a_{1}\right), N\left(a_{2}\right)\right)$.

Para continuar, se define $F$ en morfismos así: sea $f: N \longrightarrow N^{\prime}$ en rep $\mathcal{B}$, dado por la familia doble: $f=\left(f_{i}^{0}, 1 \leq i \leq 4 ; f^{1}(x), f^{1}(y)\right)$. Se construye un morfismo $g: M=F(N) \longrightarrow M^{\prime}=F\left(N^{\prime}\right)$, en $\bmod A$ mediante la familia doble: $\left(g_{1}, g_{2}, g_{4}\right)$, en donde $g_{4}=f_{4}$, y $g_{1}, g_{2}$ se definen como las matrices de bloques:

$$
g_{1}=\left(\begin{array}{cc}
f_{1}^{0} & f^{1}(x) \\
0 & f_{3}^{0}
\end{array}\right) \quad, \quad g_{2}=\left(\begin{array}{cc}
f_{3}^{0} & f^{1}(y) \\
0 & f_{2}^{0}
\end{array}\right) .
$$

Para ver que $g$ es morfismo, basta observar los siguientes dos hechos:

$$
\begin{aligned}
g_{2} M(\alpha)-M^{\prime}(\alpha) g_{1} & =\left(\begin{array}{cc}
f_{3}^{0} & f^{1}(y) \\
0 & f_{2}^{0}
\end{array}\right)\left(\begin{array}{cc}
0 & 1 \\
0 & 0
\end{array}\right)-\left(\begin{array}{cc}
0 & 1 \\
0 & 0
\end{array}\right)\left(\begin{array}{cc}
f_{1}^{0} & f^{1}(x) \\
0 & f_{3}^{0}
\end{array}\right) \\
& =\left(\begin{array}{cc}
0 & f_{3}^{0} \\
0 & 0
\end{array}\right)-\left(\begin{array}{cc}
0 & f_{3}^{0} \\
0 & 0
\end{array}\right)=0 ; \\
g_{4} M(a)-M^{\prime}(a) g_{1} & =g_{4}\left(N\left(a_{1}\right), N\left(a_{2}\right)\right)-\left(N^{\prime}\left(a_{1}\right), N^{\prime}\left(a_{2}\right)\right)\left(\begin{array}{cc}
f_{1}^{0} & f^{1}(x) \\
0 & f_{3}^{0}
\end{array}\right) \\
& =\left(g_{4} N\left(a_{1}\right)-N^{\prime}\left(a_{1}\right) f_{1}^{0}, g_{4} N\left(a_{2}\right)-N^{\prime}\left(a_{1}\right) f^{1}(x)-N^{\prime}\left(a_{2}\right) f_{3}^{0}\right) \\
& =(0,0) .
\end{aligned}
$$

La comprobación de que $F$ es efectivamente un funtor se deja al lector.

Para concluir que $F$ es una equivalencia, falta mostrar que es fiel, pleno y denso. Para la densidad, sea $M=\left(M_{1}, M_{2}, M_{4} ; M(a), M(\alpha)\right) \in \bmod A$, y considérense las descomposiciones $M_{1} \cong \operatorname{ker} M(\alpha) \oplus i m M(\alpha), M_{2} \cong i m M(\alpha) \oplus W$. Es claro que $M \cong$ $F(N)$, en donde $N \in \operatorname{rep} \mathcal{B}$ se define como: $N_{1}=\operatorname{ker} M(\alpha)$,

$N_{3}=i m M(\alpha), N_{2}=W, N_{4}=M_{4}$.

Para ver que $F$ es pleno, sea $g: M \cong F(N) \longrightarrow M^{\prime} \cong F\left(N^{\prime}\right)$, tal que $g=\left(g_{i}^{0}, 1 \leq\right.$ $\left.i \leq 4 ; g^{1}(x), g^{1}(y)\right)$. Fácilmente se muestra que con respecto a las descomposiciones (10), las transformaciones $g_{1}^{0}, g_{2}^{0}$ admiten expresiones como (15). Es fácil demostrar ahora que la familia doble: $f=\left(f_{i}^{0}, i=1,2,3, f_{4}^{0}=g_{4}^{0} ; f^{1}(x), f^{1}(y)\right)$ define un morfismo $f: N \longrightarrow N^{\prime}$, tal que $g=F(f)$. También se ve rápidamente que $F$ es fiel. 


\section{Los algoritmos}

Aquí se utiliza la palabra algoritmo como sinónimo de proceso. En la práctica, se tiene una bigráfica diferencial $(\mathcal{B}, \delta, k)$, a la cual se le aplica una transformación, obteniéndose una nueva bigráfica $\left(\mathcal{B}^{\prime}, \delta^{\prime}, k\right)$, cuyas categorías de representaciones guardan una estrecha vinculación. El paso $\mathcal{B} \rightsquigarrow \mathcal{B}^{\prime}$ da origen a un funtor fiel y pleno $F: \operatorname{rep} \mathcal{B}^{\prime} \longrightarrow$ rep $\mathcal{B}$, que permite entonces considerar la categoría rep $\mathcal{B}^{\prime}$ como una subcategoría plena de rep $\mathcal{B}$. En algunos casos, $F$ es una equivalencia, como se vio en el ejemplo de la reducción de una flecha en la sección 3.

En la literatura se han estudiado básicamente cuatro algoritmos, de los cuales se examinarán aquí solamente tres: eliminación de objetos, regularización y reducción de una flecha, dejando por fuera el 'unravelling'[cfr. Bo]. La aplicación reiterada, y hecha de manera conveniente, de estos tres algoritmos, permitirá obtener interesantes resultados para bigráficas 'schurian'.

A continuación se examina cada algoritmo, prestando atención al comportamiento de la norma de una representación y de la forma cuadrática de la bigráfica, frente a los mismos.

Dada la bigráfica diferencial triangular $(\mathcal{B}, \delta, k)$, supóngase que sus vértices son: $X_{i}, i=$ $1, \ldots, r ;$ sus flechas de grado $0: a_{j}, j=1, \ldots, n$;y sus flechas de grado $1: v_{l}, l=1, \ldots, m$. Se dirá que la bigráfica posee la estratificación $\left(a_{j} ; v_{l}\right)$. Para cada par de vértices, $X_{i}, X_{j}$, se denota por $m_{i j}\left(n_{i j}\right.$, respectivamente) el número de flechas de grado 0 (de grado 1 , respectivamente) que hay entre ellos, en cualquier sentido. La norma de una representación $M$ de $\mathcal{B}$ se define como: $\|M\|=\sum_{i, j=1}^{r} \operatorname{dim} M_{i} \operatorname{dim} M_{j}$.

La forma cuadrática de $\mathcal{B}$ se define como la aplicación $q_{\mathcal{B}}: \mathbb{Z}^{r} \longrightarrow \mathbb{Z}$, dada por

$$
q_{\mathcal{B}}\left(x_{1}, \ldots, x_{r}\right)=\sum_{i=1}^{r} x_{i}^{2}-\sum_{i, j=1}^{r} m_{i j} x_{i} x_{j}+\sum_{i, j=1}^{r} n_{i j} x_{i} x_{j}
$$

\subsection{Regularización.}

Sea $(\mathcal{B}, \delta, k)$ una bigráfica diferencial triangular con estratificación $\left(a_{j} ; v_{l}\right)$, y supóngase que $\delta\left(a_{1}\right)=v_{1}$. Se define una nueva bigráfica $\mathcal{B}_{a_{1}, v_{1}}^{\prime}$, con los mismos vértices de $\mathcal{B}$; sus flechas son las de $\mathcal{B}$, salvo que se eliminan $a_{1}$ y $v_{1}$. Es claro que se tiene una inclusión de anillos $\iota: k \mathcal{B}_{a_{1}, v_{1}}^{\prime} \longrightarrow k \mathcal{B}$. Se define un homomorfismo de anillos $t_{0}:(k \mathcal{B})_{0} \longrightarrow\left(k \mathcal{B}^{\prime}\right)_{0}$, poniendo en los generadores del primero: $t_{0}\left(e_{i}\right)=e_{i} ; t_{0}\left(a_{1}\right)=0, t_{0}\left(a_{i}\right)=a_{i}, i \geq 2$. También se define un homomorfismo de $(k \mathcal{B})_{0}$-bimódulos, $t_{1}:(k \mathcal{B})_{1} \longrightarrow\left(k \mathcal{B}_{a_{1}, v_{1}}^{\prime}\right)_{1}$, poniendo en los generadores: $t_{1}\left(v_{1}\right)=0, t_{1}\left(v_{j}\right)=v_{j}, j \geq 2$.

Tales homomorfismos inducen un homomorfismo de anillos $t: k \mathcal{B} \longrightarrow k \mathcal{B}_{a_{1}, v_{1}}^{\prime}$ [BZ, §4]. Sea ahora $\delta^{\prime}=t \delta \iota: k \mathcal{B}_{a_{1}, v_{1}}^{\prime} \longrightarrow k \mathcal{B}_{a_{1}, v_{1}}^{\prime}$. Fácilmente se demuestra que $\delta^{\prime}$ es una diferencial para $\mathcal{B}_{a_{1}, v_{1}}^{\prime}$. Así, por ejemplo, obsérvese que $\left(\delta^{\prime}\right)^{2}=t \delta(\iota t) \delta \iota=t(\delta \iota) \delta \iota=t \delta^{2} \iota=0$.

La relación de la diferencial $\delta^{\prime}$ con la diferencial original es clara: para una flecha $a \neq a_{1}$, de grado 0 en $\mathcal{B}$ con diferencial $\delta(a)=\sum_{l} b_{l} x_{l} c_{l}$, se tiene: $\delta^{\prime}(a)=\sum_{l \neq 1 ; b_{l}, c_{l} \notin\left\langle a_{1}\right\rangle} b_{l} x_{l} c_{l}$. Una fórmula silmilar es válida para las flechas de grado 1 . Se dice que la bigráfica diferencial $\left(\mathcal{B}^{\prime}, \delta^{\prime}, k\right)$ se obtiene de $(\mathcal{B}, \delta, k)$ por regularización. 
4.1.1 Teorema. Si $\left(\mathcal{B}^{\prime}, \delta^{\prime}, k\right)$ se obtiene de $(\mathcal{B}, \delta, k)$ por regularización, entonces existe una equivalencia de categorías $F: \operatorname{rep}\left(\mathcal{B}^{\prime}, \delta^{\prime}, k\right) \stackrel{\sim}{\longrightarrow} \operatorname{rep}(\mathcal{B}, \delta, k)$.

Demostración. Para $N \in \operatorname{rep} \mathcal{B}^{\prime}$, se define $F(N)=M \in \operatorname{rep} \mathcal{B}$ así: $M_{i}=N_{i} ; M\left(a_{1}\right)=$ $0, \quad M\left(a_{j}\right)=N\left(a_{j}\right), j \geq 2$. Para un morfismo $f^{\prime}: N \longrightarrow N^{\prime}$ en $\mathcal{B}^{\prime}, f^{\prime}=\left(\left(f^{\prime}\right)_{i}^{0} ;\left(f^{\prime}\right)^{1}\left(v_{j}\right), j \geq\right.$ $2)$, se define un morfismo $f: M \longrightarrow M^{\prime}$, como $f=\left(\left(f^{\prime}\right)_{i}^{0} ; f^{1}\left(v_{1}\right)=0, f^{1}\left(v_{j}\right)=\right.$ $\left.\left(f^{\prime}\right)^{1}\left(v_{j}\right), j \geq 2\right)$. Para ver que $f$ es efectivamente un morfismo, basta observar que para toda $a: X_{s} \longrightarrow X_{t}$ de grado 0 en $\mathcal{B}$, se tiene: $f_{t}^{0} M(a)-M^{\prime}(a) f_{s}^{0}=f_{t}^{0} N(a)-N^{\prime}(a) f_{s}^{0}=$ $f^{\prime}\left(\delta^{\prime}(a)\right)=f(\delta(a))$. Se define: $f=F\left(f^{\prime}\right)$. Es fácil ver que se ha construido un funtor $F: r e p \mathcal{B}^{\prime} \longrightarrow \operatorname{rep} \mathcal{B}$.

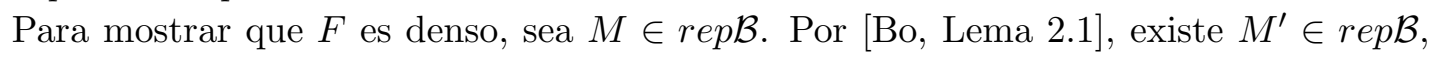
tal que $M^{\prime}\left(a_{1}\right)=0, M^{\prime} \cong M$. Se define $N \in \operatorname{rep\mathcal {B}^{\prime }}$ así: $N_{i}=M_{i}^{\prime}, 1 \leq i \leq r ; N\left(a_{j}\right)=$ $M^{\prime}\left(a_{j}\right), 2 \leq i \leq m$. Es claro que $F(N)=M^{\prime} \cong M$.

Ahora se comprobará que $F$ es pleno. Sea $f: M \longrightarrow M^{\prime}$ un morfismo en rep $\mathcal{B}$, $M=F(N), M^{\prime}=F\left(N^{\prime}\right)$, puede suponerse [Bo, Lema 2.1], que $M\left(a_{1}\right)=M^{\prime}\left(a_{1}\right)=0, \mathrm{y}$ $f^{1}\left(v_{1}\right)=0$. Considérese la familia doble $f^{\prime}=\left(f_{i}^{0} ;\left(f^{\prime}\right)^{1}\left(v_{j}\right)=f^{1}\left(v_{j}\right), j \geq 2\right)$. Entonces $f^{\prime}: N \longrightarrow N^{\prime}$ es morfismo en rep $\mathcal{B}^{\prime}$. En efecto, sea $a: X_{s} \longrightarrow X_{t}$ flecha de grado 0 en $\mathcal{B}$, necesariamente $a \neq a_{1}$. Por ser $f$ es morfismo, y ya que $f^{\prime}\left(\delta^{\prime}(a)\right)=f(\delta(a))$ se tiene: $\left(f^{\prime}\right)_{t}^{0} N(a)-N^{\prime}(a)\left(f^{\prime}\right)_{s}^{0}=f_{t}^{0} M(a)-M^{\prime}(a) f_{s}^{0}=f(\delta(a))=f^{\prime}\left(\delta^{\prime}(a)\right)$. Para terminar, es claro que $F$ es fiel.

Para determinar el comportamiento de la norma y la forma cuadrática ante la regularización, obsérvese que se pierde una flecha de grado 1 al cambiar de categoría, lo cual puede afectar la norma; pero la forma cuadrática no varía, puesto que se eliminan dos flechas de distintos grados entre los mismos vértices. El resultado preciso se establece a continuación.

4.1.2 Teorema. Bajo las condiciones del Teorema 4.1.1, supóngase que $a_{1}: X_{i} \longrightarrow$ $X_{j}, v_{1}: X_{i}-\rightarrow X_{j}$ y se regulariza. Entonces para $M=F(N)$, se tiene: $\|N\|=\|M\|-$ $\operatorname{dim} M_{i} \operatorname{dim} N_{j}$. En particular, si $M$ es sincera, i.e. $\operatorname{dim} M_{i} \neq 0$, para todos los $i$, entonces $\|N\|<\|M\|$. Para las formas cuadráticas de las bigráficas se verifica : $q_{\mathcal{B}^{\prime}}(\underline{\operatorname{dim}} N)=$ $q_{\mathcal{B}}(\underline{\operatorname{dim}} M)$.

\subsection{Eliminación de objetos}

Así como el algoritmo anterior borra flechas 'superfluas', el siguiente elimina vértices 'no deseados'. La estrategia es similar al caso anterior: se realiza cierta transformación de bigráficas $\mathcal{B} \rightsquigarrow \mathcal{B}^{\prime}$, obteniéndose un funtor fiel y pleno

rep $\mathcal{B}^{\prime} \longrightarrow$ rep $\mathcal{B}$. Incluso la técnica es parecida.

Sea $(\mathcal{B}, \delta, k)$ una bigráfica diferencial triangular con vértices $X_{1}, \ldots, X_{r}$ y estratificación $\left(a_{j} ; v_{l}\right)$. Sea $X_{l}$ un vértice arbitrario. Se define una bigráfica $\mathcal{B}_{l}^{\prime}$ con los mismos vértices de $\mathcal{B}$, salvo $X_{l}$. Por definición, las flechas de $\mathcal{B}_{l}^{\prime}$ son las de $\mathcal{B}$, excepto aquellas con algún extremo en $X_{l}$. El conjunto de las flechas de grado 0 de $\mathcal{B}_{l}^{\prime}$ se denota por $C$; el de aquellas de grado 1 , por $T$.

El álgebra $A^{\prime}=\left(k \mathcal{B}_{l}^{\prime}\right)_{0}$ es subálgebra de $A=(k \mathcal{B})_{0}$, y además se tiene una inmersión de $A^{\prime}$-subbimódulos $\left(k \mathcal{B}_{l}^{\prime}\right)_{1} \subset(k \mathcal{B})_{1}$, los cuales inducen una inmersión de anillos $\iota$ : 
$k \mathcal{B}_{l}^{\prime} \hookrightarrow k \mathcal{B}$. A continuación se define un homomorfismo de anillos $t: k \mathcal{B} \longrightarrow k \mathcal{B}_{l}^{\prime}$, que será inducido por el par $\left(t_{0}, t_{1}\right)$, par que se construye a continuación. En primer lugar, $t_{0}:(k \mathcal{B})_{0} \longrightarrow\left(k \mathcal{B}_{l}^{\prime}\right)_{0}$ es el homomorfismo de anillos tal que: $e_{l} \mapsto 0, e_{i} \mapsto e_{i}, i \neq l ; a_{i} \mapsto$ 0 , si $a_{i} \notin C, a_{i} \mapsto a_{i}$, si $a_{i} \in C$. Por otra parte, el morfismo de $A^{\prime}$-bimódulos $t_{1}$ : $(k \mathcal{B})_{1} \longrightarrow\left(k \mathcal{B}_{l}^{\prime}\right)_{1}$ se define por: $v_{i} \mapsto 0$, si $v_{i} \notin T, v_{i} \mapsto v_{i}$, si $v_{i} \in T$.

La diferencial de la nueva bigráfica se define como $\delta^{\prime}=t \delta \iota: k \mathcal{B}_{l}^{\prime} \longrightarrow k \mathcal{B}_{l}^{\prime}$, siendo la comprobación de que es realmente una diferencial como en la regularización. Para toda flecha $a$ de grado 0 en $\mathcal{B}_{l}^{\prime}$, si $\delta(a)=\sum_{l} b_{l} x_{l} c_{l}$, un cálculo fácil muestra que $\delta(a)=$ $\sum_{b_{l}, c_{l} \in\langle C\rangle ; x_{l} \in T} b_{l} x_{l} c_{l}$.

4.2.1 Teorema $\mathrm{Si}$ en la bigráfica $(\mathcal{B}, \delta, k)$ se elimina el vértice $X_{l}$, obteniéndose la bigráfica $\left(\mathcal{B}^{\prime}, \delta^{\prime}, k\right)$, entonces existe un funtor fiel y pleno $F: \operatorname{rep} \mathcal{B}^{\prime} \longrightarrow \operatorname{rep} \mathcal{B}$, que establece una equivalencia entre $\operatorname{rep\mathcal {B}^{\prime }}$ y la subcategoría plena de $\operatorname{rep} \mathcal{B}$, formada por las representaciones $M$ tales que $M_{l}=0$. Además, si $M \cong F(N)$, entonces $\|N\|=\|M\|$, y $q_{\mathcal{B}^{\prime}}(\underline{\operatorname{dim}} N)=q_{\mathcal{B}}(\underline{\operatorname{dim}} M)$.

Demostración. Para $N \in \operatorname{rep} \mathcal{B}^{\prime}$, se define $F(N)=M \in \operatorname{rep} \mathcal{B}$ así: $M_{i}=N_{i}, i \neq$ $l, M_{l}=0$. Para un morfismo $f^{\prime}: N \longrightarrow N^{\prime}$ en rep $\mathcal{B}^{\prime}$, dado por $f^{\prime}=\left(\left(f^{\prime}\right)_{i \neq l}^{0} ;\left(f^{\prime}\right)^{1}\left(v_{j}\right), v_{j} \in\right.$ $T)$, se pone $f=F\left(f^{\prime}\right)=\left(\left(f^{\prime}\right)_{i \neq l}^{0}, f_{l}^{0}=0 ; f^{1}\left(v_{j}\right)=\left(f^{\prime}\right)^{1}\left(v_{j}\right), v_{j} \in T, f^{1}\left(v_{j}\right)=0, v_{j} \notin T\right)$. Se demuestra sin dificultad que $f$ es morfismo en repB, y que $F$ es funtor. De manera similar a como se hizo para la regularización, puede mostrarse que $F$ es pleno y fiel. Es claro que $M_{l}=0$. Para terminar con la primera afirmación del teorema, obsérvese que

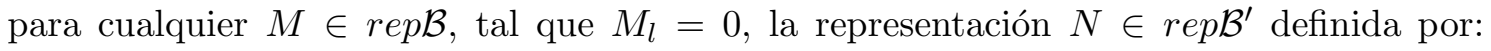
$N_{i}=M_{i}, i \neq l ; N\left(a_{i}\right)=M\left(a_{i}\right), a_{i} \in C$, verifica: $M=F(N)$.

Para la segunda afirmación, si $M=F(N)$, entonces en la suma $\|M\|=\sum_{i, j=1}^{r} \operatorname{dim} M_{i} \operatorname{dim} M_{j}$ intervienen únicamente los índices $i$ tales que $M_{i} \neq 0$, luego $\|M\|=\|N\|$. La igualdad sobre las formas cuadráticas se muestra de manera parecida.

El algoritmo de 'eliminación de objetos' se utiliza frecuentemente al inicio de ciertas demostraciones. En tales casos se procede por inducción sobre la norma de una representación, aprovechando que ésta disminuye cuando se aplica un algoritmo como la regularización o la reducción de una flecha, eso sí bajo el supuesto de que la representación es sincera, es decir, su vector dimensión tiene todas las entradas distintas de 0. Se dice: 'sea $M \in$ rep $\mathcal{B}$; puede suponerse que $M$ es sincera', pues si no lo es, se eliminan los vértices $l$ de $\mathcal{B}$ con $M_{l}=0$, obteniéndose una bigráfica $\mathcal{B}^{\prime}$, y un funtor fiel y pleno $F:$ rep $\mathcal{B}^{\prime} \hookrightarrow$ rep $\mathcal{B}$. La representación $N \in$ rep $\mathcal{B}^{\prime}$, tal que $M \cong F(N)$ es sincera y verifica: $\|M\|=\|N\|$. Si ahora se reduce una flecha en $\mathcal{B}^{\prime}$, con funtor $G: \operatorname{rep} \mathcal{B}^{\prime} \longrightarrow$ rep $\mathcal{B}^{\prime \prime}$, entonces $\|G(N)\|<\|N\|$, y puede aplicarse la hipótesis inductiva.

\subsection{Reducción de una flecha}

Sea $(\mathcal{B}, \delta, k)$ una bigráfica diferencial triangular con vértices $X_{1}, \ldots, X_{r}$ y estratificación $\left(a_{j} ; v_{l}\right)$. Si se tiene una flecha de grado $0, \alpha: X_{i} \longrightarrow X_{j}, i \neq j, \delta(\alpha)=0$, ésta puede reducirse, por el proceso que se aplicó en la sección 3. Sólo que allá la bigráfica inicial es un carcaj, el cual constituye una bigráfica sin flechas de grado 1 y con diferencial nula, motivo 
por el cual ahora se explicará este algoritmo en general. Los detalles de las demostraciones se obviarán, tomando en cuenta que las ideas fundamentales ya se expusieron ampliamente en la sección 3.

Por notación, se supondrá que los vértices son: $X_{1}, X_{2}, X_{4}, \ldots, X_{r}$ (ninguno se llama $\left.X_{3}\right)$ y que $\alpha: X_{1} \longrightarrow X_{2}$. Los vértices de la nueva bigráfica $\mathcal{B}^{\prime}$ son: $X_{1}, X_{2}, X_{3}, X_{4}, \ldots, X_{r}$ (se introdujo uno nuevo: $X_{3}$ ).

Se definen dos flechas de grado 1 en $\mathcal{B}^{\prime}: X_{1} \leftarrow \stackrel{x}{-}-X_{3} \leftarrow \stackrel{y}{-}-X_{2}$.

Las flechas de grado 0 en $\mathcal{B}^{\prime}$ son las siguientes: Toda flecha $a: X_{p} \longrightarrow X_{q}$ de grado 0 en $\mathcal{B}, a \neq \alpha$, determina 1,2 ó 4 flechas de grado 0 en $\mathcal{B}^{\prime}$, de acuerdo a la siguiente regla:

(1) si $p, q \neq 1,2$, se tiene una flecha $a_{11}: X_{p} \longrightarrow X_{q}$; (2.1) si $a: X_{1} \longrightarrow X_{q}, q \neq 1,2$, entonces se tienen 2 flechas $: a_{11}: X_{1} \longrightarrow X_{q}, a_{12}: X_{3} \longrightarrow X_{q} ;(2.2)$ si $a: X_{p} \longrightarrow X_{1}$, $p \neq 1,2$, se tienen : $a_{11}: X_{p} \longrightarrow X_{1}, a_{21}: X_{p} \longrightarrow X_{3} ;(3.1)$ todo lazo $a: X_{1} \longrightarrow X_{1}$ determina 4 flechas: $a_{11}: X_{1} \longrightarrow X_{1}, a_{12}: X_{3} \longrightarrow X_{1}, a_{21}: X_{1} \longrightarrow X_{3}, a_{22}: X_{3} \longrightarrow X_{3}$; (3.2) similarmente, todo lazo $a: X_{3} \longrightarrow X_{3}$, define 4 flechas: $a_{11}: X_{3} \longrightarrow X_{3}, a_{12}: X_{2} \longrightarrow$ $X_{3}, a_{21}: X_{3} \longrightarrow X_{2}, a_{22}: X_{2} \longrightarrow X_{2} ;(4.1)$ toda $a: X_{1} \longrightarrow X_{2}, a \neq \alpha$, define 4 flechas $: a_{11}: X_{1} \longrightarrow X_{3}, a_{12}: X_{3} \longrightarrow X_{3}, a_{21}: X_{1} \longrightarrow X_{2}, a_{22}: X_{3} \longrightarrow X_{2} ;$ (4.2) similarmente, para $a: X_{2} \longrightarrow X_{1}$, se tienen 4 flechas : $a_{11}: X_{3} \longrightarrow X_{1}, a_{12}: X_{2} \longrightarrow X_{1}, a_{21}: X_{3} \longrightarrow$ $X_{3}, a_{22}: X_{2} \longrightarrow X_{3}$.

Las flechas de grado 1 en $\mathcal{B}^{\prime}$, además de las ya definidas $x, y$, se obtienen a partir de cada flecha $v: X_{p}--\rightarrow X_{q}$, de grado 1 en $\mathcal{B}$, según la regla recién explicada para las flechas de grado 0 .

Se utilizará de ahora en adelante la siguiente notación matricial para las flechas de $\mathcal{B}^{\prime}$, según los casos anteriores :

(1) $A_{a}=\left(a_{11}\right) ;(2.1) A_{a}=\left(a_{11}, a_{12}\right), \quad(2.2) A_{a}=\left(\begin{array}{c}a_{11} \\ a_{21}\end{array}\right)$;

$(3.1,3.2,4.1,4.2) A_{a}=\left(a_{i j}\right), 1 \leq i, j \leq 2$.

Sea $e_{3}$ el idempotente en $X_{3}$; se define la matriz $A_{\alpha}=\left(\begin{array}{cc}0 & e_{3} \\ 0 & 0\end{array}\right)$.

De la misma manera, se construye una matriz $A_{v}$, para cada flecha $v$ de grado 1 en $\mathcal{B}$.

Para un camino $\gamma=\gamma_{1} \ldots \gamma_{s}$ en $\mathcal{B}$, con $\operatorname{gr}\left(\gamma_{i}\right) \in\{0,1\}$, se define la matriz $A_{\gamma}=$ $A_{\gamma_{1}} \ldots A_{\gamma_{s}}$; y para una combinación lineal de caminos $u=\sum_{i} c_{i} \gamma_{i}$, se define la matriz : $A_{u}=\sum_{i} c_{i} A_{\gamma_{i}}$.

También se introduce, para todo vértice $X_{p}$ en $\mathcal{B}$, la matriz $Y_{p}$, dada por :

$$
Y_{1}=\left(\begin{array}{cc}
0 & x \\
0 & 0
\end{array}\right), Y_{2}=\left(\begin{array}{ll}
0 & y \\
0 & 0
\end{array}\right), Y_{p}=0 \text {, si } p \neq 1,2
$$

Para una flecha $a_{s}$ de grado 0 en $\mathcal{B}$, se utiliza también la notación matricial $A_{a_{s}}=\left(a_{i j}^{(s)}\right)$, con los índices en los rangos adecuados. Para matrices $X=\left(x_{i j}\right), Y=\left(y_{i j}\right)$ con entradas en $k \mathcal{B}^{\prime}, X Y$ denotará el producto usual de matrices con entradas en dicho anillo. Una matriz $X=\left(x_{i j}\right)$ con entradas en $k \mathcal{B}^{\prime}$ se llama homogénea si todas sus entradas $x_{i j}$ tienen el mismo grado; en este caso se escribe : $\operatorname{gr} X=\operatorname{gr}\left(x_{i j}\right)$. También se define $\operatorname{gr} Y_{i}=1$, $i=1,2$. 
4.3.1 Definición. Para toda flecha $a: X_{p} \longrightarrow X_{q}$ en $\mathcal{B}, \operatorname{gr}(a)=0, a \neq \alpha$, se define la matriz $\delta^{\prime}\left(A_{a}\right)=Y_{q} A_{a}-A_{a} Y_{p}+A_{\delta(a)}$. Asimismo, para $v: X_{p}-\rightarrow X_{q}$ en $\mathcal{B}, \operatorname{gr}(v)=1$, se define la matriz $\delta^{\prime}\left(A_{v}\right)=Y_{q} A_{v}+A_{v} Y_{p}+\sum_{s} A_{v_{2}} A_{v_{s_{1}}}$, en donde $\delta(v)=\sum_{s} v_{s_{2}} v_{s_{1}}$. También se define : $\delta^{\prime}\left(A_{\alpha}\right)=0, \delta^{\prime}\left(Y_{l}\right)=Y_{l}^{2}$.

Para matrices homogéneas $X, Y$ es válida la regla de Leibnitz: $\delta^{\prime}(X Y)=\delta^{\prime}(X) Y+(-1)^{g r(X)} X \delta^{\prime}(Y)$. En efecto, para $X=\left(x_{i j}\right), Y=\left(y_{i j}\right)$, se tiene :

$$
\begin{aligned}
\left(\delta^{\prime}(X) Y+(-1)^{g r(X)} X \delta^{\prime}(Y)\right)_{i j} & =\sum_{l} \delta^{\prime}\left(x_{i l}\right) y_{l j}+\sum_{l}(-1)^{g r x_{i l}} x_{i l} \delta^{\prime}\left(y_{l j}\right) \\
& =\sum_{l} \delta^{\prime}\left(x_{i l}\right) y_{l j}+(-1)^{g r} x_{i l} x_{i l} \delta^{\prime}\left(y_{l j}\right) \\
& =\sum_{l} \delta^{\prime}\left(x_{i l} y_{l j}\right)=\delta^{\prime}\left(\sum_{l} x_{i l} y_{l j}\right) \\
& =\delta^{\prime}\left((X Y)_{i j}\right)=\left(\delta^{\prime}(X Y)\right)_{i j} .
\end{aligned}
$$

4.3.2 Lema. $\left(\delta^{\prime}\right)^{2}=0$.

Demostración. Por la regla de Leibnitz, es suficiente demostrar que $\left(\delta^{\prime}\right)^{2}\left(A_{a}\right)=$ $0,\left(\delta^{\prime}\right)^{2}\left(A_{v}\right)=0$, con $g r(a)=0, g r(v)=1$. Se hará sólo el primer caso, el otro es parecido [cfr. Bo]. Sea $a: X_{p} \longrightarrow X_{q}, a \neq \alpha$

$$
\begin{aligned}
\left(\delta^{\prime}\right)^{2}\left(A_{a}\right)= & \delta^{\prime}\left(Y_{q} A_{a}-A_{a} Y_{p}+A_{\delta(a)}\right) \\
= & \delta^{\prime}\left(Y_{q}\right) A_{a}-Y_{q} \delta^{\prime}\left(A_{a}\right)-\delta^{\prime}\left(A_{a}\right) Y_{p}- \\
& -A_{a} \delta^{\prime}\left(Y_{p}\right)+\delta^{\prime}\left(A_{\delta(a)}\right) \\
= & \left(Y_{q} Y_{q}\right) A_{a}-Y_{q}\left(Y_{q} A_{a}\right)+ \\
& +Y_{q} A_{a} Y_{p}-Y_{q} A_{\delta(a)}-Y_{q} A_{a} Y_{p}+ \\
& +A_{a}\left(Y_{p} Y_{p}\right)-A_{\delta(a)} Y_{p}- \\
& -A_{a} Y_{p} Y_{p}+\delta^{\prime}\left(A_{\delta(a)}\right) \\
= & -Y_{q} A_{\delta(a)}-A_{\delta(a)} Y_{p}+\delta^{\prime}\left(A_{\delta(a)}\right) \\
= & 0
\end{aligned}
$$

puesto que de $\delta(\delta(a))=0$, se sigue que $\delta^{\prime}\left(A_{\delta(a)}\right)=Y_{q} A_{\delta(a)}+A_{\delta(a)} Y_{p}$. Esta última afirmación no es del todo inmediata, [cfr. Bo].

4.4.3 Definición. Las diferenciales de $a_{i j}^{(s)}$ y de $v_{i j}^{(t)}$ se definen como las entradas de las matrices $\delta^{\prime}\left(A_{a_{s}}\right), \delta^{\prime}\left(A_{v_{t}}\right)$. Es decir,

$$
\delta^{\prime}\left(A_{a_{s}}\right)=\left(\delta^{\prime}\left(a_{i j}^{(s)}\right)\right), \delta^{\prime}\left(A_{v_{t}}\right)=\left(\delta^{\prime}\left(v_{i j}^{(t)}\right)\right) .
$$

Obsérvese que de $Y_{l}^{2}=0$, se sigue $\delta^{\prime}\left(Y_{l}\right)=0$, y entonces : $\delta^{\prime}(x)=\delta^{\prime}(y)=0$.

De la regla de Leibnitz para matrices, se deduce que la misma es válida para flechas de $\mathcal{B}^{\prime}$, es decir, si $a, b$ son flechas en $\mathcal{B}^{\prime}$, entonces $\delta^{\prime}(a b)=\delta^{\prime}(a) b+(-1)^{\text {gra }} b$. 
Utilizando dicha regla, se extiende $\delta^{\prime}$ a caminos (finitos) en $k \mathcal{B}^{\prime}$, y luego por linealidad a todo $k \mathcal{B}^{\prime}$. Del Lema 4.3 , se deduce que $\delta^{\prime}: k \mathcal{B}^{\prime} \longrightarrow k \mathcal{B}^{\prime}$ verifica $\left(\delta^{\prime}\right)^{2}=0$. Se ha demostrado entonces la proposición siguiente.

4.3.4 Proposición. $\delta^{\prime}$ define una diferencial en $\mathcal{B}^{\prime}$.

A continuación se establecerá una equivalencia : $F: \operatorname{rep\mathcal {B}^{\prime }} \longrightarrow$ rep $\mathcal{B}$.

Para un objeto $N \in \operatorname{rep\mathcal {B}^{\prime }}, N=\left(N_{i}, i=1,2,3,4, \ldots r ; N\left(a_{i j}^{(s)}\right), s=1,2, \ldots, n\right)$, se define $M=F(N) \in \operatorname{rep} \mathcal{B}$ así: $M_{1}=N_{1} \oplus N_{3}, M_{2}=N_{3} \oplus N_{2}, M_{l}=N_{l}, l \geq 4$.

Además, se definen las transformaciones $M(\alpha): M_{1} \longrightarrow M_{2}, M\left(a_{s}\right)$ por las matrices :

$$
M(\alpha)=\left(\begin{array}{cc}
0 & i d_{N_{3}} \\
0 & 0
\end{array}\right), M\left(a_{s}\right)=\left(N\left(a_{i j}^{(s)}\right)\right) .
$$

Si se tiene $f: N \longrightarrow N^{\prime}$ en $r e p \mathcal{B}^{\prime}, f=\left(f_{i}^{0}, i=1,2,3, \ldots, r ; f^{1}(x), f^{1}(y), f^{1}\left(v_{i j}^{(s)}\right), s=\right.$ $1,2, \ldots m)$, se define un morfismo $g: M=F(N) \longrightarrow F\left(N^{\prime}\right)=M^{\prime}$ en repB $\mathcal{B}$, como $g=$ $\left(g_{i}^{0}, i=1,2,4, \ldots, r ; g^{1}\left(v_{t}\right)\right)$, en donde

$$
\begin{aligned}
g_{1}^{0} & =\left(\begin{array}{cc}
f_{1}^{0} & f^{1}(x) \\
0 & f_{3}^{0}
\end{array}\right), \quad g_{2}^{0}=\left(\begin{array}{cc}
f_{3}^{0} & f^{1}(y) \\
0 & f_{2}^{0}
\end{array}\right), \\
g_{l}^{0} & =f_{l}^{0}, l \geq 4, g^{1}\left(v_{t}\right)=\left(f^{1}\left(v_{i j}^{(t)}\right)\right) .
\end{aligned}
$$

Se demuestra que $g$ es un morfismo, procediendo como en la sección 3.

4.3.5 Proposición. $F$ es una equivalencia de categorías.

Demostración. Para ver que $F$ es denso, dada $M \in \operatorname{rep}(\mathcal{B})$, la transformación lineal $M(\alpha): M_{1} \longrightarrow M_{2}$ induce descomposiciones: $M_{1} \cong \operatorname{ker} M(\alpha) \oplus i m M(\alpha), M_{2}=$ $\operatorname{im} M(\alpha) \oplus W$, con $W \subseteq M_{2}$. Se define $N \in \operatorname{rep}\left(\mathcal{B}^{\prime}\right)$ mediante : $N_{1}=\operatorname{ker} M(\alpha), N_{3}=$ $i m M(\alpha), N_{2}=W, N_{l}=M_{l}, l \geq 4$; además, toda $M\left(a_{s}\right)$ se expresa, con respecto a las descomposiciones en sumas directas anteriores, como una matriz $M\left(a_{s}\right)=\left(M_{i j}\left(a_{s}\right)\right)$, de 1,2 ó 4 entradas. Para toda flecha $a_{i j}^{(s)}$ de grado 0 en $\mathcal{B}^{\prime}$, se define la transformación lineal : $N\left(a_{i j}^{(s)}\right)=M_{i j}\left(a_{s}\right)$.

Se ha construido así una representación $N \in$ rep $\mathcal{B}^{\prime}$, y fácilmente se comprueba que $M \cong F(N)$.

Para ver que $F$ es pleno, sea $g: M \cong F(N) \longrightarrow F\left(N^{\prime}\right) \cong M^{\prime}$ un morfismo, dado por : $g=\left(g_{i}^{0}, i=1,2,4, \ldots, r ; g^{1}\left(v_{t}\right), t=1, \ldots, m\right)$.

Se tienen descomposiciones :

$$
\begin{aligned}
& M_{1} \cong \operatorname{ker} M(\alpha) \oplus i m M(\alpha), M_{2}=i m M(\alpha) \oplus W, \\
& M_{1}^{\prime} \cong \operatorname{ker} M^{\prime}(\alpha) \oplus i m M^{\prime}(\alpha), M_{2}^{\prime}=i m M^{\prime}(\alpha) \oplus W^{\prime},
\end{aligned}
$$

con respecto a las cuales, como se prueba fácilmente, $g_{1}^{0}, g_{2}^{0}$ admiten expresiones matriciales :

$$
g_{1}^{0}=\left(\begin{array}{cc}
A_{11} & A_{12} \\
0 & A_{22}
\end{array}\right), g_{2}^{0}=\left(\begin{array}{cc}
B_{11} & B_{12} \\
0 & B_{22}
\end{array}\right) .
$$


Además, de la condición $\delta(\alpha)=0$, se deduce : $B_{11}=A_{22}$. Se introduce una nueva notación para las entradas de estas matrices :

$$
g_{1}^{0}=\left(\begin{array}{cc}
f_{1}^{0} & f^{1}(x) \\
0 & f_{3}^{0}
\end{array}\right), g_{2}^{0}=\left(\begin{array}{cc}
f_{3}^{0} & f^{1}(y) \\
0 & f_{2}^{0}
\end{array}\right) .
$$

Sea $f=\left(f_{1}^{0}, f_{3}^{0}, f_{2}^{0}, f_{l}^{0}=g_{l}^{0}, l \geq 4 ; f^{1}(x), f^{1}(y), f^{1}\left(v_{i j}^{(t)}\right), t=1, \ldots, m\right)$, en donde las $f^{1}\left(v_{i j}^{(t)}\right)$ están definidas por : $g^{1}\left(v_{t}\right)=\left(f^{1}\left(v_{i j}^{(t)}\right)\right)$.

Se demostrará que se tiene un morfismo $f: N \longrightarrow N^{\prime}$. En efecto, para toda flecha de grado $0, a: X_{p} \longrightarrow X_{q}$, en $\mathcal{B}, a \neq \alpha$, se tiene : $g_{q}^{0} M(a)-M^{\prime}(a) g_{p}^{0}=g(\delta(a))$.

Se sabe que : $g_{q}^{0} M(a)-M^{\prime}(a) g_{p}^{0}=f\left(\delta^{\prime}\left(A_{a}\right)\right)-f\left(Y_{q} A_{a}-A_{a} Y_{p}\right)$, y además, se verifica : $\left(g_{q}^{0} M(a)-M^{\prime}(a) g_{p}^{0}+f\left(Y_{q} A_{a}-A_{a} Y_{p}\right)\right)_{i j}=f_{e}^{0} N\left(a_{i j}\right)-N^{\prime}\left(a_{i j}\right) f_{s}^{0}$, en donde $a_{i j}: X_{s} \longrightarrow X_{e}$.

Se tiene entonces : $f_{e}^{0} N\left(a_{i j}\right)-N^{\prime}\left(a_{i j}\right) f_{s}^{0}=f\left(\delta^{\prime}\left(a_{i j}\right)\right)$, así que $f$ es morfismo. Es claro que $g=F(f)$.

Es fácil ver que el funtor $F$ es fiel.

Las aplicaciones se basan en el control de la variación de la norma y de la forma cuadrática frente a cada algoritmo. Los teoremas 4.2.2 y 4.3.1 dan cuenta de los casos de la regularización y la eliminación de objetos. A continuación se estudia lo relativo a la reducción.

4.3.6 Teorema En las condiciones del apartado 4.4, si se reduce la flecha $\alpha$, y $M \cong$ $F(N)$, con $\underline{\operatorname{dim}} N=\left(x_{1}, x_{3}, x_{2}, x_{4}, \ldots\right), \underline{\operatorname{dim}} M=\left(x_{1}+x_{3}, x_{3}+x_{2}, x_{4}, \ldots\right)$, entonces $\|N\|=$ $\|M\|-\left(x_{1}+x_{3}\right)\left(x_{3}+x_{2}\right)$, de donde, $\|N\| \leq\|M\|$. En particular, si $M$ es sincera, la desigualdad anterior es estricta.

Además, $q_{\mathcal{B}^{\prime}}(\underline{\operatorname{dim}} N)=q_{\mathcal{B}}(\underline{\operatorname{dim}} M)+x_{1} x_{2}$.

Demostración. Para cada par de vértices $i, j$ de $\mathcal{B}$, se tiene :

$$
\begin{aligned}
\|M\|= & m_{12}\left(x_{1}+x_{3}\right)\left(x_{3}+x_{2}\right)+m_{11}\left(x_{1}+x_{3}\right)^{2}+ \\
& +m_{22}\left(x_{3}+x_{2}\right)^{2}+\sum_{p=4}^{n} m_{1 p}\left(x_{1}+x_{3}\right) x_{p}+ \\
& +\sum_{p=4}^{n} m_{2 p}\left(x_{3}+x_{2}\right) x_{p}+\sum_{p, q=4}^{n} m_{p q} x_{p} x_{q} .
\end{aligned}
$$

Según la definición de la bigráfica $\mathcal{B}^{\prime}$, se tiene :

$$
\begin{aligned}
\|N\|= & \left(m_{12}-1\right)\left(x_{1} x_{2}+x_{1} x_{3}+x_{3} x_{2}+x_{3}^{2}\right)+ \\
& m_{11}\left(x_{1}^{2}+2 x_{1} x_{3}+x_{3}^{2}\right)+m_{22}\left(x_{3}^{2}+2 x_{3} x_{2}+x_{2}^{2}\right) \\
& +\sum_{p=4}^{n} m_{1 p}\left(x_{1} x_{p}+x_{3} x_{p)}+\sum_{p=4}^{n} m_{2 p}\left(x_{3} x_{p}+x_{2} x_{p}\right)\right. \\
& +\sum_{p, q=4}^{n} m_{p q} x_{p} x_{q} \\
= & \|M\|-\left(x_{1}+x_{3}\right)\left(x_{3}+x_{2}\right) .
\end{aligned}
$$


Para la segunda aseveración, obsérvese que

$$
q_{\mathcal{B}}(\underline{\operatorname{dim}} M)=\left(x_{1}+x_{3}\right)^{2}+\left(x_{3}+x_{2}\right)^{2}+\sum_{p=4}^{n} x_{p}^{2}-\|M\|+q_{\mathcal{B}}^{(1)}(\underline{\operatorname{dim}} M)
$$

en donde

$$
\begin{aligned}
q_{\mathcal{B}}^{(1)}(\underline{\operatorname{dim}} M)= & n_{11}\left(x_{1}+x_{3}\right)^{2}+n_{22}\left(x_{3}+x_{2}\right)^{2}+ \\
& +n_{12}\left(x_{1}+x_{3}\right)\left(x_{3}+x_{2}\right)+ \\
& +\sum_{p=4}^{n} n_{1 p}\left(x_{1}++x_{3}\right) x_{p}+\sum_{p=4}^{n} n_{2 p}\left(x_{3}+x_{2}\right) x_{p}+ \\
& +\sum_{p, q=4}^{n} n_{p q} x_{p} x_{q}
\end{aligned}
$$

Por otra parte,

$$
q_{\mathcal{B}}(\underline{\operatorname{dim}} N)=\sum_{p=1}^{n} x_{p}^{2}-\|N\|+q_{\mathcal{B}^{\prime}}^{(1)}(\underline{\operatorname{dim}} N)
$$

con

$$
\begin{aligned}
q_{\mathcal{B}^{\prime}}^{(1)}(\underline{\operatorname{dim} N)=} & x_{1} x_{3}+x_{3} x_{2}+n_{12}\left(x_{1} x_{2}+x_{1} x_{3}+x_{3} x_{2}+x_{3}^{2}\right)+ \\
& +n_{11}\left(x_{1}^{2}+2 x_{1} x_{3}+x_{3}^{2}\right)+n_{22}\left(x_{3}^{2}+2 x_{3} x_{2}+x_{2}^{2}\right)+ \\
& +\sum_{p=4}^{n} n_{1 p}\left(x_{1} x_{p}+x_{3} x_{p}\right)+\sum^{n} n_{2 p}\left(x_{3} x_{p}+x_{2} x_{p}\right)+ \\
& +\sum_{p, q=4}^{n} n_{p q} x_{p} x_{q} .
\end{aligned}
$$

De lo anterior se deduce el resultado anunciado :

$$
\begin{aligned}
q_{\mathcal{B}^{\prime}}(\underline{\operatorname{dim}} N)-q_{\mathcal{B}}(\underline{\operatorname{dim}} M)= & x_{1}^{2}+x_{3}^{2}+x_{2}^{2}-\left(x_{1}+x_{3}\right)^{2}-\left(x_{3}+x_{2}\right)^{2}+ \\
& +(\|M\|-\|N\|)+ \\
& +\left(q_{\mathcal{B}^{\prime}}^{(1)}(\underline{\operatorname{dim}} N)-q_{\mathcal{B}}^{(1)}(\underline{\operatorname{dim}} M)\right) \\
= & \left(-x_{3}^{2}-2 x_{1} x_{3}-2 x_{2} x_{3}\right)+\left(x_{1}+x_{3}\right)\left(x_{3}+x_{2}\right)+ \\
& +\left(x_{1} x_{3}+x_{2} x_{3}\right) \\
= & x_{1} x_{2}
\end{aligned}
$$

Una consecuencia inmediata es que si se aplica alguno de los algoritmos: $\mathcal{B} \rightsquigarrow \mathcal{B}^{\prime}$, entonces 'la forma cuadrática no disminuye', i.e. para $M \cong F(N), q_{\mathcal{B}^{\prime}}(\underline{\operatorname{dim}} N) \geq q_{\mathcal{B}}(\underline{\operatorname{dim}} M)$. 


\section{Bigráficas 'schurian'}

Los algoritmos de la sección anterior se aplican aquí a las bigráficas schurian, las cuales se caracterizan por carecer de lazos. De fundamental importancia es el control que se tiene sobre la variación de la norma y de la forma cuadrática al aplicar algún algoritmo. Como se verá, la disminución de la norma permite argumentar reiteradamente por inducción, en tanto que el comportamiento de la forma cuadrática da pie para utilizar enfoques combinatorios. Ejemplos importantes de bigráficas schurian son los conjuntos parcialmente ordenados de tipo de representación finito [S].

Si $(\mathcal{B}, \delta, k)$ es una bigráfica diferencial y $M$ una representación, todo $t \in k$ determina un endomorfismo $\bar{t}$ de $M$, dado por la familia doble $\bar{t}=\left(\bar{t}_{i}^{0} ; 0\right)$, tal que $\bar{t}_{i}^{0}: M_{i} \longrightarrow M_{i}, m \mapsto t m$. Para $a: i \longrightarrow j$, flecha de grado 0 , es claro que $\bar{t}(\delta(a))=0, \mathrm{y}$ además, $\left(\bar{t}_{j}^{0} M(a)-M(a) \bar{t}_{i}^{0}\right)(m)=0=\bar{t}(\delta(a))$, para todo $m \in M_{i} \quad ;$ luego $\bar{t}$ es morfismo. Los morfismos de este tipo se llaman 'diagonales'. Interesa destacar el caso en que las representaciones inescindibles no poseen más endomorfismos que los diagonales.

5.1 Definición. Una bigráfica diferencial $(\mathcal{B}, \delta, k)$ es de tipo 'schurian' si para toda representación inescindible $M, \operatorname{End}_{\mathcal{B}}(M) \cong k$.

5.2 Lema. Una bigráfica schurian no posee lazos de ningún grado.

Demostración Por contradicción, si existe un lazo de grado $0, \alpha: l \longrightarrow l$, se tiene una representación inescindible $M_{\alpha}$, dada por $\left(M_{\alpha}\right)_{l}=k^{2},\left(M_{\alpha}\right)_{i}=0, i \neq l$, y para toda flecha $a: i \longrightarrow j,\left(M_{\alpha}\right)(\alpha)=\left(\begin{array}{ll}0 & 1 \\ 0 & 0\end{array}\right) ; M_{\alpha}(a)=0, a \neq \alpha$. La familia doble $f=\left(f_{i}^{0} ; f^{1}(x)=0\right)$, con $f_{1}^{0}: M_{l} \longrightarrow M_{l}, f_{1}^{0}=\left(\begin{array}{ll}0 & 1 \\ 0 & 0\end{array}\right), f_{i}^{0}=0$, si $i \neq l$, es un morfismo, como se comprueba fácilmente. Pero este morfismo no es escalar, imposible.

(ii) De nuevo por contradicción, si existe un lazo $x: l--\rightarrow l$, de grado 1 , considérese la representación simple $S_{l}$. Se define $\widetilde{x}: S_{l} \longrightarrow S_{l}$ por $\widetilde{x}_{1}^{0}: k \longrightarrow k, \widetilde{x}_{1}^{0}=i d_{k}, \widetilde{x}_{i}^{0}=$ $0, i \neq l ; \widetilde{x}^{1}(x)=i d_{k}, \widetilde{x}^{1}(y)=0, y \neq x$. Para ver que $\widetilde{x}$ es morfismo, obsérvese que para toda flecha $a$ de grado $0, \widetilde{x}(\delta(a))=0$. En efecto, si $\delta(a)=\sum_{t} b_{t} x_{t} c_{t}$, entonces $\widetilde{x}(\delta(a))=\sum_{t} \lambda_{t} S_{l}\left(b_{t}\right) \widetilde{x}^{1}\left(x_{t}\right) S_{l}\left(c_{t}\right)=0$, pues en cada sumando alguno de los tres factores es nulo. Puesto que $\widetilde{x}$ no es morfismo escalar, se tiene una contradicción

5.3 Lema Sea $S_{l}$ una representación simple de una bigráfica diferencial semisimple $\mathcal{B}$, y sean $x_{1}, \ldots, x_{t}$ la totalidad de los lazos de grado 1 en el vértice $l$. Entonces $\operatorname{dim}_{k} \operatorname{End}_{\mathcal{B}}\left(S_{l}\right)=t+1$.

Demostración Si $t=0$, el resultado es claro. Si $t \geq 1$, sea $\widetilde{x}_{i}$ el endomorfismo definido por $x_{i}$, según (ii) de la demostración de (2.2), $1 \leq i \leq t$. Se tiene entonces una $k$-base $\left\{i d, \widetilde{x}_{1}, \ldots, \widetilde{x}_{t}\right\}$. En efecto, un $f \in \operatorname{End}_{\mathcal{B}}\left(S_{l}\right)$ está dado por una familia doble $f=\left(a_{0} ; a_{1}, \ldots, a_{t}\right)$, los $a_{i} \in k$. Si $a_{0}=0, f=\sum a_{i} \widetilde{x}_{i}$; si $a_{0} \neq 0$, entonces $f=a_{0}^{-1} i d+$ $\sum_{i} a_{0}^{-1} a_{i} \widetilde{x}_{i}$. Es clara la independencia lineal

5.4 Teorema Si $\mathcal{B}$ es bigráfica diferencial triangular schurian y su forma cuadrática es débilmente positiva, entonces para toda representación inescindible $M, \underline{\operatorname{dim}} M$ es raíz 
positiva de $q_{\mathcal{B}}$, es decir, $q_{\mathcal{B}}(\underline{\operatorname{dim}} M)=1$. Además, toda representación inescindible está determinada por su vector dimensión. En consecuencia, $\mathcal{B}$ es de tipo de representación finito.

Demostración (i) Sea $M$ una representación inescindible. Se probará que $q_{\mathcal{B}}(\underline{\operatorname{dim}} M)=$ 1 , por inducción sobre $\|M\|$. Puede suponerse que $M$ es sincera, sin pérdida de generalidad. Si $\|M\|=0$, entonces la bigráfica $\mathcal{B}$ no posee flechas de grado 0, luego sus únicas representaciones inescindibles son las simples, así que $M$ es simple. Si los vértices de $\mathcal{B}$ son $X_{1}, \ldots, X_{n}$, y dado que ella es schurian, su forma cuadrática es $q_{\mathcal{B}}\left(x_{1}, \ldots, x_{n}\right)=\sum_{i=1}^{n} x_{i}^{2}$. Luego, $q_{\mathcal{B}}(\underline{\operatorname{dim}} M)=1$.

Sea $\|M\|>0$, y supóngase cierta la afirmación para toda representación inescindible $N$, en cualquier bigráfica, con $\|N\|<\|M\|$. Sea $a: i \longrightarrow j$ una flecha de grado 0 , con $\delta(a)$ mínima. Necesariamente $i \neq j$. Si $\delta(\alpha)=0$, se reduce $a$, obteniéndose una bigráfica $\mathcal{B}^{\prime}$ y una equivalencia $F: \operatorname{rep}\left(\mathcal{B}^{\prime}\right) \longrightarrow \operatorname{rep}(\mathcal{B})$, y tomando $M=F(N)$, se tiene $\|N\|<\|M\|$. Entonces $q_{\mathcal{B}^{\prime}}(\underline{\operatorname{dim}} N)=1$, y como $q_{\mathcal{B}}$ es débilmente positiva, $0<$ $q_{\mathcal{B}}(\underline{\operatorname{dim}} M) \leq q_{\mathcal{B}^{\prime}}(\underline{\operatorname{dim}} N)=1$, luego $q_{\mathcal{B}}(\underline{\operatorname{dim}} M)=1$. Si $\delta(\alpha) \neq 0, \delta(\alpha)=c_{1} v_{1}+\ldots+c_{m} v_{m}$ , con los $c_{i}$ escalares y las $v_{i}$ flechas de grado 1 . Por medio de un cambio de base, puede suponerse $\delta(\alpha)=v_{1}$, y entonces regularizar. Se procede como antes, y se obtiene la conclusión.

(ii) Sea $M$ inescindible, y supóngase que para una $M^{\prime}$ inescindible, $\underline{\operatorname{dim}} M^{\prime}=\underline{\operatorname{dim}} M$. Se probará que entonces $M^{\prime} \cong M$. Se realiza inducción sobre $\|M\|$, para lo cual puede suponerse s.p.g. que $M$ es sincera. Si $\|M\|=0$, la bigráfica es semisimple, y aquí es clara la conclusión.

En caso de que $\|M\|>0$, tómese $a: i \longrightarrow j$, con $\delta(a)$ mínima. Si $\delta(\alpha)=0$, se reduce $\alpha$ y entonces existen representaciones inescindibles $N, N^{\prime}$ de $\mathcal{B}^{\prime} \operatorname{con} M=F(N), M^{\prime}=F\left(N^{\prime}\right)$, $\|N\|<\|M\|,\left\|N^{\prime}\right\|<\left\|M^{\prime}\right\| . \operatorname{Si} \underline{\operatorname{dim}} N=\left(x_{1}, x_{2}, x_{3}, \ldots\right), \underline{\operatorname{dim}} N^{\prime}=\left(x_{1}^{\prime}, x_{2}^{\prime}, x_{3}^{\prime}, \ldots\right)$, se sabe que $x_{1} x_{2}=q_{\mathcal{B}^{\prime}}(\underline{\operatorname{dim}} N)-q_{\mathcal{B}}(\underline{\operatorname{dim}} M)=1-1=0$; igualmente se llega a: $x_{1}^{\prime} x_{2}^{\prime}=0$. Se prueba , por una combinación de los casos : $x_{1}=0, x_{2}=0, x_{1}^{\prime}=0, x_{2}^{\prime}=0$, que $\underline{\operatorname{dim}} N^{\prime}=\underline{\operatorname{dim}} N$, y entonces, por la hipótesis inductiva, $N^{\prime} \cong N$, de donde $\left.M=F(N) \cong \overline{F\left(N^{\prime}\right.}\right)=\overline{M^{\prime}}$.

Si $\delta(\alpha) \neq 0$, se puede regularizar, después de un cambio de base adecuado, y obtener $M=F(N), M^{\prime}=F\left(N^{\prime}\right),\|N\|<\|M\|,\left\|N^{\prime}\right\|<\left\|M^{\prime}\right\|$, para los cuales se cumple : $\underline{\operatorname{dim}} N=\underline{\operatorname{dim}} M=\underline{\operatorname{dim}} M^{\prime}=\underline{\operatorname{dim}} N^{\prime}$, luego $N \cong N^{\prime}$ y de aquí, $M \cong M^{\prime}$.

Para concluir, obsérvese que por ser $q_{\mathcal{B}}$ forma cuadrática débilmente positiva, por el Lema de Drozd [Ri, p.3], ella posee sólo un número finito de raíces positivas. Luego el número de vectores dimensión de inescindibles es finito, y como en cada dimensión hay solamente una clase de isomorfía, la bigráfica es de tipo de representación finito.

Es sabido $[\mathrm{RK}]$ que toda bigráfica diferencial de tipo finito tiene forma cuadrática débilmente positiva. Así, la Proposición anterior es un recíproco parcial a tal afirmación.

5.5 Proposición Si $\mathcal{B}$ es una bigráfica diferencial schurian, entonces para toda representación inescindible $M$, existe únicamente un número finito de clases de isomorfía de representaciones inescindibles $N$, tales que $\underline{\operatorname{dim}} N=\underline{\operatorname{dim}} M$.

Demostración Sea $M$ representación inescindible. Se procede por inducción sobre $\|M\|$. Si $\|M\|=0$, y dado que se puede suponer spg que $M$ es sincera, entonces la bigráfica $\mathcal{B}$ es semisimple, y en este caso, se verifica la afirmación. 
Para continuar, sea $\|M\|>0$, y supóngase cierta la afirmación para toda representación inescindible $N$, tal que $\|N\|<\|M\|$. Existe $a: i \longrightarrow j$, de grado 0 , con $\delta(a)$ mínima. Por hipótesis, $i \neq j$, y se dan dos casos.

Si $\delta(a)=0$, se reduce $a$, y para el funtor $F, M=F(N)$. Hay que demostrar que hay sólo un número finito de inescindibles $M^{\prime}$, tales que $\underline{\operatorname{dim}} M^{\prime}=\underline{\operatorname{dim}} M$. Sea $M^{\prime}=F\left(N^{\prime}\right)$. Fácilmente se concluye que $\underline{\operatorname{dim}} N^{\prime}=\underline{\operatorname{dim}} N$, entonces para $N^{\prime}$ hay sólo un número finito de posibilidades, luego para $M^{\prime}=F\left(N^{\prime}\right)$ hay sólo un número finito de posibilidades, y se ha concluido.

Si $\delta(a) \neq 0$, se regulariza y se llega fácilmente a la conclusión

5.6 Teorema Si $\mathcal{B}$ es bigráfica diferencial triangular schurian y su forma cuadrática es débilmente positiva, entonces para toda representación inescindible $M, q_{\mathcal{B}}(\underline{\operatorname{dim}} M)=1$, es decir, $\underline{\operatorname{dim}} M$ es raíz positiva de $q_{\mathcal{B}}$. Además, existe una biyección entre la familia de las clases de isomorfía de representaciones inescindibles y el conjunto de las raíces positivas de la forma cuadrática $q_{\mathcal{B}}$, dada por $M \leftrightarrow \underline{\operatorname{dim}} M$.

Demostración Por la Proposición (2.4), la aplicación $M \mapsto \underline{\operatorname{dim}} M$ está bien definida y es inyectiva. Para la sobreyectividad, obsérvese que existe una secuencia finita de bigráficas

$$
\mathcal{B}=\mathcal{B}^{(0)} \sim \mathcal{B}^{(1)} \sim \mathcal{B}^{(2)} \sim \cdots \sim \mathcal{B}^{(s)}
$$

tales que $\mathcal{B}^{(i)}$ se obtiene de $\mathcal{B}^{(i-1)}$ por regularización o por reducción de una flecha, y además, $\mathcal{B}^{(s)}$ es semisimple. Se tienen entonces equivalencias

$$
\operatorname{rep}\left(\mathcal{B}^{(s)}\right) \stackrel{\sim}{\longrightarrow} \operatorname{rep}\left(\mathcal{B}^{(s-1)}\right) \stackrel{\sim}{\longrightarrow} \cdots \operatorname{rep}\left(\mathcal{B}^{(1)}\right) \stackrel{\sim}{\longrightarrow} \operatorname{rep}(\mathcal{B})
$$

Se efectúa inducción sobre $s-i$.Si $s-i=0$, entonces $i=s$, y si $\mathcal{B}^{(s)}$ tiene $m$ vértices, entonces $q_{\mathcal{B}^{(s)}}\left(x_{1}, \ldots, x_{m}\right)=\sum_{i=1}^{m} x_{i}^{2}$, así las raíces de $q_{\mathcal{B}^{(s)}}$ corresponden biyectivamente a las clases de isomorfía de inescindibles.

Para continuar, es suficiente demostrar que si la afirmación cierta para $\mathcal{B}^{(s-i)}$, entonces lo es para $\mathcal{B}^{(s-i-1)}$. Hay dos casos. Si $F: \operatorname{rep}\left(\mathcal{B}^{(s-i)}\right) \stackrel{\sim}{\longrightarrow} \operatorname{rep}\left(\mathcal{B}^{(s-i-1)}\right)$ corresponde a una regularización, sea $\underline{d}$ tal que $q_{\mathcal{B}^{(s-i-1)}}(\underline{d})=1$. Entonces $q_{\mathcal{B}^{(s-i)}}(\underline{d})=1$, y por hipótesis, existe $N$ inescindible de $\mathcal{B}^{(s-i)}$, tal que $\underline{d}=\underline{\operatorname{dim}} N$; para $M=F(N)$ se tiene entonces $\underline{\operatorname{dim}} M=\underline{\operatorname{dim}} N=\underline{d}$.

Si $F$ corresponde a la reducción de una flecha, sea $\underline{d}=\left(d_{1}, d_{2}, d_{4, \ldots}\right)$ con $q_{\mathcal{B}^{(s-i-1)}}(\underline{d})=1$ .Se construye una raíz $\underline{d}^{\prime}$ para $q_{\mathcal{B}(s-i)}$ así : si $d_{1}>\bar{d}_{2}$, sea $\underline{d}^{\prime}=\left(d_{1}-d_{2}, d_{2}, 0, d_{4}, \ldots\right)$; si $d_{1}=d_{2}$, sea $\underline{d}^{\prime}=\left(0, d_{1}, 0, \ldots\right)$ y si $d_{1}<d_{2}$ sea $\underline{d}^{\prime}=\left(d_{2}-d_{1}, d_{1}, 0, \ldots\right)$. Se cumple $q_{\mathcal{B}^{(s-i)}}\left(\underline{d}^{\prime}\right)=q_{\mathcal{B}^{(s-i-1)}}(\underline{d})+0=q_{\mathcal{B}^{(s-i-1)}}(\underline{d})$. Por hipótesis inductiva, existe un inescindible $N$, tal que $\underline{d}^{\prime}=\underline{\operatorname{dim}} N$, y entonces el inescindible $M=F(N)$ verifica $\underline{d}=\underline{\operatorname{dim}} M$ 


\section{Referencias}

[ARS] Auslander, M.; Reiten, I.; Smalo, S. (1995) Representation Theory of Artin Algebras. Cambridge Univ. Press, Cambridge.

[BCS] Bautista, R.; Colavita, L.; Salmerón, L. (1981) "On Adjoint Funtors in Representation Theory", in Representations of Algebras, Lecture Notes in Mathematics 903, Springer-Verlag: 9-25.

[BK] Bautista, R.; Kleiner, M. (1990) "Almost split sequences for relatively projective modules", J. Algebra 135: 19-56.

[Bo] Boza, J. (1996) Algoritmos de Reducción en la Teoría de Representaciones de Coálgebras. Tesis doctoral, UNAM, México.

[BZ] Bautista, R.; Zuazúa, R. (1996) "Morita equivalence and reduction algorithms for representations of coalgebras", Canadian Math. Soc., Conference Proc. 18: 51-80.

[CB 1 Crawley-Boevey, W.W. (1988) "On tame algebras and bocses", Proc. London Math. Soc. 56(3): 451-483.

[CB 1 Crawley-Boevey, W.W. (1992) "Modules of finite length over their endomorphism rings", in Representations of Algebras and Related Topics, Cambridge Univ. Press, No. 168: 127-184.

$\left[\mathrm{CB}_{3}\right]$ Crawley-Boevey, W.W. "Matrix reductions for Artinian rings, and an application to rings of finite representation type', Journal of Algebra 157(1): 1-25.

[D $\mathrm{D}_{1}$ Drozd, Y.A. (1980) "Tame and wild matrix problems", in Representation Theory II, Lecture Notes in Mathematics 832, Springer-Verlag: 242-258.

$\left[\mathrm{D}_{2}\right]$ Drozd, Y.A. (1986) "Tame and wild matrix problems", Amer. Math. Soc. Transl. 128(2): 31-55.

[D $\left.D_{3}\right]$ Drozd, Y.A. (1992) "Matrix problems, small reduction and representations of a class of mixed Lie groups", in Representations of Algebras and Related Topics, Cambridge Univ. Press, No. 168: 225-249.

[K] Kleiner, M. (1984) "Matrix problems and representations of finite dimensional algebras", Proceed. IV-ICRA, Carleton Univ., Ottawa.

[MacL] MacLane, S. (1988) "Categories for the Working Mathematician". SpringerVerlag.

[M] Montaño, G. (1993) "Caracterización de bocses de dimensión finita de tipo manso". Tesis de Maestría, UNAM, México.

[O] Ovsienko, S.A. (1993) "Generic representations of free bocses", Preprint 93-03, Universität Bielefeld, 28 p. 
[Ri] Ringel, C.M. (1984) Tame Algebras and Integral Quadratic Forms. Lecture Notes in Mathematics, 1099, Springer-Verlag.

[Ro] Roiter, A.V. (1980) "Matrix problems and representations of bocs's", in Lecture Notes in Mathematics 831, Springer-Verlag: 288-324.

[RK] Roiter, A.V.; Kleiner, M. "Representations of differential graded categories", in Lecture Notes in Mathematics 488, Springer-Verlag: 316-339.

[S] Simson, D. (1992) "Linear representations of partially ordered sets and vector space categories", Gordon and Breach, Switzerland. 